\title{
Rational Rotation Numbers for Maps of the Circle
}

\section{Grzegorz Świątek}

Institute of Mathematics, Department of Mathematics, PKiN, 9-th floor, PL-00-901 Warsaw, Poland

\begin{abstract}
We consider families of maps of the circle of degree 1 which are homeomorphisms but not diffeomorphisms, that is maps like

$$
x \rightarrow x+t+\frac{c}{2 \pi} \sin (2 \pi x)(\bmod 1)
$$

with $c=1$. We prove that the set of parameter values corresponding to irrational rotation numbers has Lebesgue measure 0 . In other words, the intervals on which frequency-locking occurs fill up the set of full measure.
\end{abstract}

\section{Introduction and Formulation of the Theorem}

We study one-parameter families of maps of the circle of degree 1, which are differentiable homeomorphisms, but not diffeomorphisms. The subject of our investigation is dependence of rotation number on the parameter. The problem interested both mathematicians and physicists. The families like $x \rightarrow x$ $+\frac{c}{2 \pi} \sin (2 \pi x)+t$ were studied for various constants $c$. For $c<1$ the maps are diffeomorphisms and there is a result of [2], which says that rotation number is absolutely continuous as function of $t$. When $c>1$ the maps are nonhomeomorphisms and have no rotation number. However, there is a result of [6] about endpoints of rotation interval. It is quite different from the result for diffeomorphism just mentioned above -- both endpoints of rotation interval are rational almost everywhere in the sense of Lebesgue measure. The case of $c=1$ is covered by the present work. It was also studied numerically - the results strongly suggested that rotation number should be rational almost everywhere (see [3]).

We give a mathematical proof of this. In many aspects it is the continuation of [6]. Our main analytic tool is cross-ratio. Given four points $a<b<c<d$ on the real line, we define their cross-ratio by

$$
\operatorname{Cr}(a, b, c, d)=\frac{(b-a)(d-c)}{(c-a)(d-b)}
$$


Suppose also that $h: \mathbb{R} \rightarrow \mathbb{R}$ is an increasing function. The distortion $D$ of the crossratio by that function is given by

$$
D(a, b, c, d ; h)=\frac{\operatorname{Cr}(h(a), h(b), h(c), h(d))}{\operatorname{Cr}(a, b, c, d)} .
$$

It is known that if $h$ has no flat critical points then it is possible to $D$ estimate the for $h$ and, which is more interesting, for large order iterates of $h$. The estimations of that kind are used in [4] and [7] and prove to be useful there. We make the general assumption that all critical points of our maps are non-flat. We shall give precise formulation of that later. We need also differentiability with respect to the parameter in order to ensure that our maps change regularly, in particular near the critical points.

Throughout all our work we shall deal with a continuous family $f$ of lifts of homeomorphisms of the circle of degree 1 . The parameter space will be denoted by $U$, so that we have $f: U \times \mathbb{R} \rightarrow \mathbb{R}$. Parameter values will be denoted by $t$, while arguments on the real line by $x$. We also refer to particular lifts, denoted by $f_{t}$ and defined by $f_{t}(x)=f(t, x)$.

Now we shall formulate 2 sets of hypotheses.

Hypothesis 1. The family $f$ is real analytic with respect to both variables and the derivative $\frac{\partial f}{\partial t}$ is positive everywhere in $U \times \mathbb{R}$. Each $f_{t}$ has at least one critical point.

Hypothesis 2. The family $f$ is of the form $f(t, x)=h(x)+r$, where the function $h: \mathbb{R} \rightarrow \mathbb{R}$ satisfies:

a) It is at least $C^{3}$.

b) It has at least one critical point and in the neighbourhood of each critical point there exists the derivative of some order vanishing nowhere in that neighbourhood.

The Claim, which holds under any of these hypotheses says that: The Lebesgue measure of the set of parameter values for which the rotation number is irrational is zero.

\section{The Technical Version of Assumptions}

Now we shall formulate the set of assumptions which are in fact a result of localisation of the hypotheses given in the previous section. Thus we shrink the parameter space $U$ to a compact interval $T$ and assume that the following conditions are satisfied:

$1^{\circ}$ There exist $m$, with $1 \leqq m<\infty$, functions $k_{i}: T \rightarrow \mathbb{R}$, of class $C^{1}$ such that $k_{i}(t)$ is always a critical point of $f_{t}$ and all critical points of $f_{t}$ modulo 1 are represented in this way. Moreover, we suppose that:

a) $k_{i}(t)$ are distinct modulo 1 for every $t$,

b) for each ithere is an open neighbourhood $V_{i}$ of the curve $\left(t, k_{i}(t)\right)$ in $T \times \mathbb{R}$ and a natural number $l_{i}$ such that $\frac{\partial^{l_{l}} f}{\partial x^{l_{l}}}$ exists, is continuous and is non-zero everywhere in $V$.

c) $\frac{\partial^{l_{1}-1} f}{\partial x^{l_{i}-1}}\left(k_{i}(t)\right)=0$ for each $i$ and $t \in T$. 
$2^{\circ}$ The function $f$ is of class $C^{3}$ with all derivatives bounded simultaneously and uniformly by a constant L.

$3^{\circ}$ There is a positive constant $\sigma$ such that $\frac{\partial f}{\partial t} \geqq \sigma$ everywhere in $T \times \mathbb{R}$.

Then we claim that

The rotation number is irrational only on a set of zero measure.

Corollary. If Hypothesis 1 or Hypothesis 2 from the previous section holds, then for any $t \in U$ there is a neighbourhood of $t$ in $U$, which, possibly with an exception of countably many points, can be covered by compact intervals on which the assumptions of the technical version are verified.

Proof. The only difficult point is to show that Hypothesis 1 of Sect. 1 implies that critical points of $f$ can be locally represented as claimed in assumptions $1^{\circ}$ of TV (the abbreviation of "the technical version"). In order to demonstrate this we fix some $t_{0}$ in $U$ and we are going to show that some neighbourhood of $t_{0}$ can be covered, except for countably many points, by intervals on which TV is satisfied. Let the critical points of $f_{t_{0}}$ be $k_{1}, \ldots, k_{m}$ with orders $l_{1}, \ldots, l_{m}$ respectively. We assume that there exist natural numbers $\lambda_{1} \leqq l_{1}, \ldots, \lambda_{m} \leqq l_{m}$ and analytic functions $z_{1}, \ldots, z_{m}$ defined on some neighbourhood $V$ of $t_{0}$ with values on the real line such that $z_{i}\left(t_{0}\right)=k_{i}$ and the derivatives $\lambda_{i}, \ldots, l_{i}$ of $f_{t}$ vanish at $z_{i}(t)$ for all $i$ between 1 and $m$ and $t$ from $V$. We also assume that in this way we obtain all the zeros of $\frac{\partial^{\lambda} f}{\partial^{\lambda_{2}} x}$ in neighbourhoods of $\left(t_{0}, k_{i}\right)$ in $U \times \mathbb{R}$ for each $i$ respectively. Observe that it is always possible to obtain such a situation for every $t$ by the implicit function theorem. We shall proceed by induction with respect to $\sum_{i=1}^{m} \lambda_{l}$. If it is $m$, which is the least possible value, then $1^{\circ}$ is evidently satisfied in some neighbourhood of $t$ (possibly finer than $V$ ). Otherwise we consider some $i$ such that $\lambda_{i}>1$. The function $\frac{\hat{o}^{\lambda_{2}-1} f}{\partial x^{\lambda_{t}}-1}\left(z_{i}(t)\right)$ is real analytic in $V$. Hence it is either 0 identically in some neighbourhood $V^{\prime}$ of $t$ or it is non-zero in $V^{+}$and $V^{-}$one-sided neighbourhoods of $t$. In the first case we can reduce $i_{i}$ by 1 , in the second case we can do the same in one-sided neighbourhoods of $t$ using the implicit function theorem. By induction we obtain easily that each point in a punctured neighbourhood of $t$ has a neighbourhood, which can be covered by intervals on which TV holds with exception of only countably many points. The corollary follows.

Observe now, that using the corollary the theorems mentioned in Sect. 1 follow immediately from the technical version of the theorem. Indeed, every point of $U$ has a neighbourhood on which the set of parameter values corresponding to irrational rotation numbers has measure zero, hence that point cannot be a density point of this set.

Now we just assume the technical version and begin the proof of its claim. The words "technical version" will be abbreviated to TV.

We begin the proof with presentation of our main technical tool. 
Cross-Ratio Inequality. Let us consider a system of fours of points of the real line covering the circle by a canonical projection. We denote the points by $a_{i}, b_{i}, c_{i}, d_{i}$ with $a_{i}<b_{i}<c_{i}<d_{i}$ for $i=1, \ldots, n$. We assume further that the intervals $\left(a_{i}, d_{i}\right)$ projected onto the circle by the canonical projection cover each point of it at most $k$ times. For every $t \in T$ there is a constant $C_{k}$, independent of $t, n$, and the choice of points such that the following inequality holds:

$$
\prod_{i=1}^{n} D\left(a_{i}, b_{i}, c_{i}, d_{i} ; f_{t}\right) \leqq C_{k} .
$$

Before we prove the inequality we shall demonstrate several lemmas.

Lemma 1. There exists such $w>0$ such that intervals $W_{i, t}=\left(k_{i}(t)-2 w, k_{i}(t)+2 w\right)$ satisfy the following conditions for every $t \in T$ and $i$ with $1 \leqq i \leqq m$ :

a) For different $i$ and fixed the intervals $W_{i, t}$ are disjoint modulo 1.

b) The Schwarzian derivative of $f_{t}$, which is defined by

$$
S f_{t}=\frac{f_{t}^{(3)}}{f_{t}^{\prime}}-\frac{3}{2} \cdot\left(\frac{f_{t}^{\prime \prime}}{f_{t}^{\prime}}\right)^{2}
$$

is negative in $W_{i, t}$.

c) There are numbers $A_{i, t}$, uniformly bounded by a constant $A$ with the property

$$
A_{i, t} \cdot\left|x-k_{i}(t)\right|^{l_{i}-1} \leqq f_{t}^{\prime}(x) \leqq 2 \cdot A_{i, t} \cdot\left|x-k_{i}(t)\right|^{l_{i}-1} \quad \text { for } \quad x \in W_{i, t} .
$$

Proof. The condition a) will be satisfied if only $w$ is small enough. Next, we fix $i$ and consider a function

$$
a_{t}(x)=\frac{1}{\left(l_{i}-1\right) !} f_{t}^{\left(l_{l}\right)}(x)
$$

The function $a_{t}(x)$ is a continuous function of 2 variables defined in $V_{i}$. We have

$$
\begin{gathered}
f_{t}^{\prime}(x)=\left(x-k_{i}(t)\right)^{l_{i}-1} \cdot a_{t}\left(x_{1}\right), \\
f_{t}^{\prime \prime}(x)=\left(l_{i}-1\right) \cdot\left(x-k_{i}(t)\right)^{l_{i}-2} \cdot a_{t}\left(x_{2}\right), \\
f_{t}^{\prime \prime \prime}(x)=\left(l_{i}-1\right) \cdot\left(l_{i}-2\right) \cdot\left(x-k_{i}(t)\right)^{l_{l}-3} \cdot a\left(x_{3}\right),
\end{gathered}
$$

where $\left|x_{j}-k_{i}(t)\right| \leqq\left|x-k_{i}(t)\right|$ for $j=1,2,3$.

Since $a_{t}\left(k_{i}(t)\right)$ is non-zero for every $t$, the condition c) follows easily from the continuity of $a_{t}$. In order to prove b) we calculate the Schwarzian derivative as follows:

$$
S f_{t}=\left(\frac{\left(l_{i}-1\right)\left(l_{i}-2\right) \cdot a_{t}\left(x_{3}\right)}{a_{t}\left(x_{1}\right)}-\frac{3}{2} \cdot \frac{\left(l_{i}-1\right)^{2} \cdot a_{t}^{2}\left(x_{2}\right)}{a_{t}^{2}\left(x_{1}\right)}\right) \cdot\left(x-k_{i}(t)\right)^{2} .
$$

Since the ratios $\frac{a_{t}\left(x_{3}\right)}{a_{t}\left(x_{1}\right)}$ as well as $\frac{a_{t}\left(x_{2}\right)}{a_{t}\left(x_{1}\right)}$ are uniformly close to 1 when $w$ is small enough, the negativity of $S f_{t}$ follows from

$$
\left(l_{i}-1\right)\left(l_{i}-2\right)<\frac{3}{2} \cdot\left(l_{i}-1\right)^{2} \text {. QED }
$$

For each $t \in T$ we consider the set

$$
E_{t}=\mathbb{R} \backslash\left(\bigcup_{i=1}^{m}\left(k_{i}(t)-w, k_{i}(t)+w\right)+\mathbb{Z}\right)
$$


and define $E=\bigcup_{t \in T}\left(\{t\} \times E_{t}\right)$. It is easy to see that $\frac{\partial f}{\partial x} \geqq \xi$ on $E$ for some positive constant $\varrho$. Indeed, $E$ is a compact set which does not contain points like $\left(t, k_{i}(t)\right)$.

Let $a, b, c$ be any three points of the real line such that $b$ lies between $a$ and $c$. Then we denote

$$
D_{h}\left(a, b, c ; f_{t}\right)=\frac{c-a}{b-a} \cdot \frac{f_{t}(b)-f_{t}(a)}{f_{t}(c)-f_{t}(a)} .
$$

Note that $D\left(a, b, c, d ; f_{t}\right)=D_{h}\left(a, b, c ; f_{t}\right) \cdot D_{h}\left(d, c, b ; f_{t}\right)$.

Lemma 2. For any $a, b, c \in \mathbb{R}$ such that the quantity $D_{h}\left(a, b, c ; f_{t}\right)$ is defined it is not greater than a constant $K_{2}$ independent of $t$.

Proof. We fix a parameter value $t$ and we are going to remove it from further notations in this proof. It will suffice to consider $D_{h}(a, b, c ; f)$ when $a<b<c$. We consider three cases:
a) $(a, c) \subset E_{t}$.
b) $(a, c) \subset W_{i}$ for some $i$.
c) Neither a) nor b).

It is very easy to resolve a) - we immediately obtain $D_{h}(a, b, c ; f) \leqq \frac{L}{\xi}$. Observe also, that c) implies $c-a>w$. Now a simple compactness argument shows that $\frac{c-a}{f(c)-f(a)}$ is bounded by an absolute constant. So does $\frac{f(b)-f(a)}{b-a}$, which is bounded by $L$. Now we shall concentrate on $b$ ).

We substitute $\mathbf{a}=a-k_{i}(t), \mathbf{b}=b-k_{i}(t), \mathbf{c}=c-k_{i}(t)$. We also denote $h_{i}(x)=x^{l_{\tau}}$. We compute

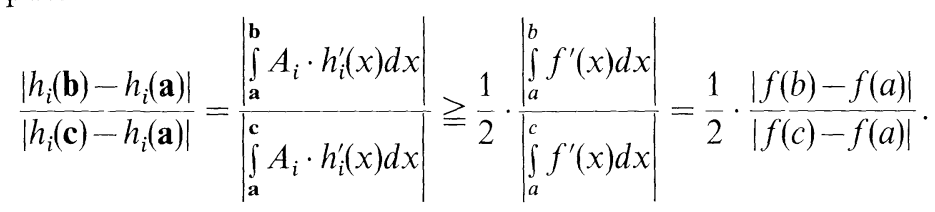

The inequality here follows by Lemma 1c). Since evidently $\frac{|c-a|}{|b-a|}=\frac{|\mathbf{c}-\mathbf{a}|}{|\mathbf{b}-\mathbf{a}|}$, it follows that $D_{h}(a, b, c ; f) \leqq 2 \cdot D_{h}\left(a, b, c ; h_{i}\right)$. Further we observe that $D_{h}\left(\mathbf{a} r, \mathbf{b} r, \mathbf{c} r ; h_{i}\right)$ $=D_{h}\left(\mathbf{a}, \mathbf{b}, \mathbf{c} ; h_{i}\right)$ for any $r$ different from 0 . Hence we may assume $\mathbf{a}=1$ and consider $D_{h}$ as a function of two variables $\mathbf{c}$ and $\mathbf{b}$. It is clear that if $|\mathbf{c}|$ is large enough (not less than $\left.l_{i}\right)$, then $D_{h}\left(1, \mathbf{b}, \mathbf{c} ; h_{i}\right)<1$. Since $D_{h}$ also has finite limits when $\mathbf{b}$ or $\mathbf{c}$ tends to 1 , it has a bound independent of both, as well as $t$, by the compactness argument. This constant may be done independent of $i$ too, by taking maximum with respect to $i$.

QED

Proof of the Cross-Ratio Inequality. We divide the set $M=\{1, \ldots, n\}$ into 3 subsets $M_{1}, M_{2}, M_{3}$ in the following way:

$1^{\circ}$ A number $i$ belongs to $M_{1}$ if and only if $\left(a_{i}, d_{i}\right)$ is contained in some $W_{j, t}$.

$2^{\circ}$ The number $i$ belongs to $M_{2}$ if and only if $\left(a_{i}, d_{i}\right)$ is contained in $E_{t}$.

$3^{\circ} M_{3}=M \backslash\left(M_{1} \cup M_{2}\right)$.

We shall prove that each of the products

$$
\prod_{i \in M_{s}} D\left(a_{i}, b_{i}, c_{i}, d_{i} ; f_{t}\right)
$$


$S=1,2,3$ is bounded by a universal constant, which will clearly yield the cross-ratio inequality.

$1^{\circ}$ We define $M_{1 a}$ a subset of $M_{1}$ in the following way:

$$
M_{1 a}=\left\{i \in M_{1}:\left(a_{i}, d_{i}\right) \text { contains a critical point of } f_{t}\right\} .
$$

Since the cardinality of this set is bounded by $m \cdot k$, we obtain by Lemma 2 that

$$
\prod_{j \in M_{1 a}} D\left(a_{i}, b_{i}, c_{i}, d_{i} ; f_{t}\right) \leqq K_{2}^{2 k m} .
$$

If $i \in M_{1} \backslash M_{1 a}$, then the Schwarzian derivative of $f_{t}$ is negative on $\left(a_{i}, d_{i}\right)$ by Lemma 1b). It is a well-known fact (see for example [5] that it implies $D\left(a_{i}, b_{i}, c_{i}, d_{i} ; f_{t}\right)<1$.

$2^{\circ}$ Since the derivative of $f$ with respect to $x$ is bounded away from 0 by $\xi$ uniformly in $E$, the variation of $\log f_{t}^{\prime}$ over $E_{t}$ is bounded by $\frac{L}{\varrho}$ for every $t$. The
classical distortion argument gives us the estimation.

for every $t$.

$$
\prod_{i \in M_{2}} D\left(a_{i}, b_{i}, c_{i}, d_{i} ; f_{t}\right) \leqq \exp \left(2 k \cdot \frac{L}{\varrho}\right)
$$

$3^{\circ}$ Since $W_{j, t}$ and $E_{t}$ overlap, $i \in M_{3}$ implies that $d_{i}-a_{i}>w$. There are at most $\frac{k}{w}$ such intervals and their contribution to the distortion of the cross-ratio can be bounded by Lemma 2 .

This concludes the proof. QED

\section{Basic Notions, Notations, and Ideas}

First we shall briefly present the formalism of Farey series. It is described in an

elementary way in [1].
A Farey interval is an interval $A=\left(\frac{p^{\prime}}{q^{\prime}}, \frac{p}{q}\right)\left(q, q^{\prime}>0\right)$ with $p q^{\prime}-q p^{\prime}=1$. We shall quote without proofs several properties of Farey intervals.

A) The rational in $A$ with smallest denominator is $\frac{p+p^{\prime}}{q+q^{\prime}}$.

B) There is no other rational in this interval with denominator less than $2 \cdot \min \left(q, q^{\prime}\right)+\max \left(q, q^{\prime}\right)$.

We shall usually work with good Farey intervals, that is Farey intervals satisfying $\max \left(q, q^{\prime}\right)<2 \cdot \min \left(q, q^{\prime}\right)$. The notations $A, p, p^{\prime}, q, q^{\prime}$ will be used in the sense given above throughout the whole work. For technical and notational simplicity we shall usually assume that $q^{\prime}>q$, which implies also $p^{\prime}>p$. We shall call such Farey interval a normalised one. We note that for $r=p^{\prime}-p$ and $s=q^{\prime}-q$ the interval $\left(\frac{r}{s}, \frac{p}{q}\right)$ is a Farey interval containing $A$. That fact holds also if $\frac{p}{q}<\frac{p^{\prime}}{q^{\prime}}$ with ordering of endpoints of the intervening Farey intervals consequently reversed.

The main subject of our interest is the function which ascribes to every parameter value $t$ the rotation number of $f_{t}$. It is denoted by $\varrho$. It is well-known that $\varrho$ is continuous and, as a consequence of the assumption $3 \mathrm{TV}$, non-decreasing in $T$. 
Now we shall briefly sketch a scheme of our proof. We fix a good normalised Farey interval $A$ and consider functions $f_{t}$ for $t \in \varrho^{-1}(\bar{A})$. Notice that the results will be valid for not normalised intervals as well because of the following trick. By an affine change of parameters we can make $T$ be the interval $[-1,1]$. We consider a family of maps $f_{t}^{\prime}$ defined by $f_{t}^{\prime}(x)=-f_{-t}(-x)$ and the corresponding function $\varrho^{\prime}$. It is easy to verify that $f^{\prime}$ satisfies the hypotheses of TV if only $f$ does so. There is also an equality $\varrho^{\prime}(t)=1-\varrho(t) \bmod 1$. Hence if $A=\left(\frac{p^{\prime}}{q^{\prime},} \frac{p}{q}\right)$ is not normalised we may consider $f_{t}^{\prime}$ for $t \in Q^{\prime-1}\left(\left[\frac{q-p}{q}, \frac{q^{\prime}-p^{\prime}}{q^{\prime}}\right]\right)$ with the last interval already normalised. Any results concerning proportion of the set of parameter values with rational rotation numbers obtained for $f^{\prime}$ on that interval imply the same estimates for $f$ on $\varrho^{-1}(\bar{A})$. Namely, we shall show that independently of $A$ the proportion of the measure of $\varrho^{-1}\left(\left\{\frac{p^{\prime}}{q^{\prime}}\right\},\left\{\frac{p}{q}\right\}\right)$ to the measure of $\varrho^{-1}(\bar{A})$ is not smaller than some positive constant. This will easily yield the claim of the technical theorem.

In Sect. 4 we begin with simple considerations concerning the link between arithmetic properties of rotation numbers, expressed in terms of Farey series formalism and ordering of orbits of the maps. In Sect. 5 we thoroughly study periodic orbits of a critical point of $f_{t}$ for various rotation numbers from $A$, namely either of the form $u_{n}=\frac{n p+p^{\prime}}{n q+q^{\prime}}$ or $v_{n}=\frac{p+n p^{\prime}}{q+n q^{\prime}}$. These sequences tend to the endpoints of $A$. We shall examine how much orbits of the critical point for those rotation numbers differ from orbits with periods $q$ and $q^{\prime}$. We measure this difference in a geometrically transparent way by taking the distances between points and their $q$-th (respectively $q^{\prime}$-th) images. The result is that the differences between periodic orbits of the critical point for rotation numbers $u_{n}$ and $\frac{p}{q}, v_{n}$ and $\frac{p^{\prime}}{q^{\prime}}$ and between $\frac{p^{\prime}}{q^{\prime}}$ and $\frac{p}{q}$ are all comparable by uniform constants, independent of $A$ and, what is more interesting, of $n$. The idea of Sect. 6 and Sect. 7 is to show that this implies that analogous distances between parameter values are also comparable, which clearly gives the desired estimation. This can be done directly though the calculations involved are fairly complicated. Section 8 is an easy conclusion.

We finish this section with some notations.

Definition 3.1. For any continuous function $z$ from the parameter space to the real line and an integer $i$ we define the function $g_{i}(\cdot ; z): T \rightarrow \mathbb{R}$ by the formula $g_{i}(t ; z)$ $=f_{t}^{i}(z(t))$.

Definition 3.2. For a function $z$ as above and a Farey interval $A$ we define points in the parameter space as follows:

$$
E(z, A)=\sup \left\{t \in T: g_{q^{\prime}}(t ; z)=p^{\prime}+z(t)\right\}
$$

and

$$
H(z, A)=\inf \left\{t \in T: g_{q}(t ; z)=p+z(t)\right\} .
$$

The interval $(E(z, A), H(z, A))$ will be denoted by $I(A ; z)$. 
The above definition makes sense if the sets mentioned are both non-empty. We assume that it is the case.

Once and for all we choose one of the functions $k_{i}$ defined in $1^{\circ} \mathrm{TV}$. We shall denote it by $k$ and refer to its values as the critical point.

\section{Rotation Numbers Determine Orbits}

We fix a good Farey interval $A=\left(\frac{p^{\prime}}{q^{\prime \prime}} \frac{p}{q}\right)$ as usual.

Lemma 3. Suppose that the function $z$ from definitions of Sect. 3 is a constant $P$. Then the intervals $g_{i}\left((E(P, A), H(P, A) ; P)\right.$ for $i \geqq 1$ and $i \leqq \max \left(q, q^{\prime}\right)$ when projected onto the circle cover each point of it at most twice.

Proof. Suppose the contrary. To simplify notations we shall remove $A$ and $P$ from them. Let $\pi$ be a canonical projection. We can find $i_{1}, i_{2}, i_{3}$ natural numbers not exceeding $q^{\prime \prime}=\max \left(q, q^{\prime}\right)$ such that

$$
\pi\left(g_{i_{1}}(H)\right) \in \pi\left(g_{i_{2}}(E, H)\right) \cap \pi\left(g_{i_{3}}(E, H)\right) .
$$

Since $f_{t}$ are increasing with $t$ it is easy to see that it implies also

$$
\pi\left(g_{i_{1}+j}(H)\right) \in \pi\left(g_{i_{2}+j}(E, H)\right) \cap \pi\left(g_{i_{3}+j}(E, H)\right)
$$

for any natural $j$. In particular, it implies that there are parameter values $t_{2}$ and $t_{3}$ in $I(A ; P)$ such that $P$ is periodic with period $i_{2}+q^{\prime \prime}-i_{1}$ and $i_{3}+q^{\prime \prime}-i_{1}$ for $f_{t_{2}}$ and $f_{t_{3}}$ respectively $\left[\right.$ if $q^{\prime \prime}=q^{\prime}$ one should choose $i_{1}, i_{2}, i_{3}$ so to have

$$
\pi\left(g_{i_{1}}(E)\right) \in \pi\left(g_{i_{2}}(I)\right) \cap \pi\left(g_{i_{3}}(I)\right)
$$

in order to obtain this corollary]. Suppose that $i_{2}+q^{\prime \prime}-i_{1}=q+q^{\prime}$, which is the least possible value, then $i_{3}+q^{\prime \prime}-i_{1}$ must be at least $q+q^{\prime}+\min \left(q, q^{\prime}\right)>2 q^{\prime \prime}$ because $A$ is a good Farey interval. We obtain a contradiction. QED

Lemma 4. Let $A$ be a Farey interval, not necessarily a good one and $\varrho(t) \in A$. Then, for any point $P$ on the circle the positively oriented $\operatorname{arcs}\left(\pi \circ f_{t}^{q} \circ \pi^{-1}(P), P\right)$ and $\left(P, \pi \circ f_{t}^{q^{\prime}} \circ \pi^{-1}(P)\right)$ contain no point of the form $\pi \circ f_{t}^{i} \circ \pi^{-1}(P)$ for $0 \leqq i<q+q^{\prime}$.

Proof. Suppose for example that there exists such $i$ as above and $\pi \circ f_{t}^{i} \circ \pi^{-1}(P)$ belongs to the second of the intervals mentioned above. Consider the family $f_{t}$ on the interval $(E(P, A), t)(P$ here means the constant function equal to $P)$. Since for $E(P, A) f^{i}(P)$ does not belong to $\left[P, f_{t}^{q}\right]$ (which degenerates to the single point), there must exist some intermediate parameter value $T$ such that either $f_{T}^{i}(P)$ $=P(\bmod 1)$ or $f_{T}^{i}(P)=f_{T}^{q^{\prime}}(P)$. In either case there is an orbit of $f_{T}$ of period less than $q+q^{\prime}$, but in $A$ there are not rotation numbers with denominators less than $q+q^{\prime} . \quad$ QED

Lemma 5. Let $\varrho(t) \in A$. We assume as usual that the endpoints of $A$ are $\frac{p}{q}$ and $\frac{p^{\prime}}{q^{\prime}}$ with $q^{\prime}-q=s$, but now instead of $\frac{p}{q}>\frac{p^{\prime}}{q^{\prime}}$ we assume that $q^{\prime}>q$. Let $P$ be any point of the circle. By I we denote the arc $\left(\pi \circ f_{t}^{q}(P), \pi \circ f_{t}^{s}(P)\right)$ and $I^{\prime}$ is $\left(\pi \circ f_{t}^{q-s}(P), f_{t}^{s}(P)\right)$. We claim 
that $\operatorname{arcs} I, \pi \circ f_{t} \circ \pi^{-1}(I), \ldots, \pi \circ f_{t}^{q-1} \circ \pi^{-1}(I)$ cover each point of the circle at most twice and so do the $\operatorname{arcs} I^{\prime}, \ldots, \pi \circ f_{t}^{s-1}\left(I^{\prime}\right)$.

Proof. In both cases it suffices to consider only points of $I$ or $I^{\prime}$ because $f_{t}$ is a homeomorphism. By Lemma 4 there is no iterate of $f_{t}$ of order less than $q^{\prime}+q$ other than $q^{\prime}$ by which the image of $P$ belongs to $I$. Thus the only iterate of order less than $q$ by which and endpoint of $I$ comes to $I$ is the $q-s^{\text {th }}$, which proves the first part of the claim. The second assertion follows in the same way when we consider a Farey interval with endpoints $\frac{r}{s}$ and $\frac{p-r}{q-s}$ and apply Lemma 4 to it. QED

Finally we shall consider the case when the function $z$ of definitions of Sect. 3 is no longer constant, but may be one of the functions $k_{i}$ defined in $1^{\circ} \mathrm{TV}$. We choose once and for all one of those functions and shall denote it by $k$.

Lemma 6. Let $z$ be either a constant function or $k$. Then, for every positive $i$ the derivative $\frac{d g_{i}(t ; z)}{d t}$ is not less than the positive constant $\sigma$ mentioned $3^{\circ} \mathrm{TV}$.

Proof. We compute for every positive $i$ :

$$
\frac{d g_{i}(t ; z)}{d t}=\frac{\partial f}{\partial t}\left(t, g_{i-1}(t ; z)\right)+\frac{\partial f}{\partial x}\left(t, g_{i-1}(t ; z)\right) \cdot \frac{d g_{i-1}(t ; z)}{d t} \text {. }
$$

In the situation of the lemma, the second term vanishes for $i=1$. The first term is then not less than $\sigma$, in particular positive. The second term is always non-negative by induction and the lemma follows. QED

A function $z$ satisfying the hypotheses of Lemma 6 will be referred to as a variable point. We introduce the notation $j(A)=\varrho^{-1}(A)$ for a good Farey interval A.

In the following proposition we shall use simplified notations. We shall remove function $z$ whenever it will be the function $k$ and for $E(z, A)$ and $H(z, A)$ we shall write just $E$ and $H$. Proposition 1. Suppose that $\frac{|j(A)|}{|I(A)|} \geqq \frac{1}{2}$. Then there is a constant $N$ independent of $A$
such that the intervals

$$
g_{1}(I(A ; k) ; k), \ldots, g_{q+s}(I(A ; k) ; k)
$$

when projected onto the circle cover each point of it at most $N$ times.

Proof. Whenever we do not specify a variable point we mean the function $k$. The notation $A$ will be also omitted. Let us choose points $P_{1}, \ldots, P_{n}$ in the closure of $I$ in such a way that $E=P_{1}<P_{2}<\ldots<P_{n}=H$. Then we consider intervals $I_{j}$, $j=1, \ldots, n$ defined by $I_{j}=I\left(A ; k\left(P_{j}\right)\right)$, where $k\left(P_{j}\right)$ is regarded as the constant variable point. We shall prove that if the points $P$ are sufficiently close to each other, then $g_{i}\left(I_{j} ; k\left(P_{j}\right)\right)$ and $g_{i}\left(I_{j+1} ; k\left(P_{j+1}\right)\right)$ intersect for every positive $i$ and $j=1, \ldots, n-1$. It means that the sets $U_{i}=\bigcup_{j=1}^{n} g_{i}\left(I_{j} ; k\left(P_{j}\right)\right)$ are all connected. Since we have assumed that $P_{1}=E$ and $P_{n}=H$, each $U_{i}$ contains both $g_{i}(E)$ and $g_{i}(H)$. Thus 
it contains the whole $g_{i}(I)$. It is a simple corollary to Lemma 3 that $U_{1}, \ldots, U_{q+s}$ cover each point of the circle at most $2 n$ times. The proof is finished by the following.

Auxiliary Lemma. Under the assumptions of Proposition 2 there exists a positive constant $\delta$, independent of $A$, such that if $P, R \in I$ and $|P-R|<\delta \cdot|I|$, then the intervals $g_{i}(I(A ; k(P)) ; k(P))$ and $g_{i}(I(A ; k(R)) ; k(R))$ intersect for every positive $i$.

Proof. We begin with the following simple observation:

$$
\varrho(H(k(P)))=\varrho(H(k(R)))=\frac{p}{q},
$$

while

$$
\varrho(E(k(P)))=\varrho(E(k(R)))=\frac{p^{\prime}}{q^{\prime}} .
$$

It implies the inequality

$$
\otimes: \min (H(k(P)), H(k(R)))-\max (E(k(P)), E(k(R))) \geqq|j(A)| .
$$

We shall prove that choosing $\delta$ appropriately we can ensure that the following inequalities hold:

$$
g_{1}(H(k(P)) ; k(P))>g_{1}(E(k(R)) ; k(R))
$$

and

$$
g_{1}(H(k(R)) ; k(R))>g_{1}(E(k(P)) ; k(P)) .
$$

We shall prove only the first one - the proof of the other is practically the same. We estimate:

$$
\begin{aligned}
& g_{1}(H(k(P)) ; k(P))-g_{1}(E(k(R)) ; k(P)) \geqq \sigma \cdot|H(k(P))-E(k(R))| \\
& \quad \geqq \sigma \cdot|j(A)| \geqq \frac{\sigma}{2} \cdot|I(A)|,
\end{aligned}
$$

where the successive inequalities follow from Lemma $6, \otimes$ and the assumption of Proposition 1. On the other hand:

$$
\begin{aligned}
& g_{1}(E(k(R)) ; k(R))-g_{1}(E(k(R)) ; k(P)) \leqq f(E(k(R)), k(R))-f(E(k(P)), k(P)) \\
& \quad \leqq L \cdot|k(R)-k(P)| \leqq B \cdot L \cdot|P-R|
\end{aligned}
$$

where we have used $2^{\circ} \mathrm{TV}$ and by $B$ we have denoted sup $\left\{\left|\frac{d k}{d t}\right|: t \in T\right\}$ which is finite provided $1^{\circ} \mathrm{TV}$ holds. Thus if $\frac{\sigma}{2} \cdot|I(A)|>B L \cdot|P-R|$, we obtain the inequality we desire. The above condition must hold if we put $\delta=\frac{\sigma}{2 B L}$.

Now we shall prove that $g_{i}(H(k(P)) ; k(P))>g_{i}(E(k(R)) ; k(R))$ for every $i$ positive integer, once more the proof of the inequality

$$
g_{i}(H(k(R)) ; k(R))>g_{i}(E(k(P)) ; k(P))
$$


is quite similar. It is a simple induction:

$$
\begin{aligned}
& g_{i+1}(E(k(R)) ; k(R))=f\left(E(k(R)), g_{i}(E(k(R)) ; k(R))\right. \\
& \quad<f\left(H(k(P)), g_{i}(H(k(P)) ; k(P))\right)=g_{i+1}(H(k(P)) ; k(P))
\end{aligned}
$$

- we have only used $\otimes$ and the fact that $f$ is increasing with respect to the first argument and non-decreasing with respect to the second one.

It is clear that the inequalities we have proved yield the claim of the lemma.

The proof of Proposition 1 is also finished - the Auxiliary Lemma ensures that for every $A$ we can choose no more than $\left[\frac{1}{\delta}\right]+2$ points $P_{i}$ with the desired properties. We get $N=2\left(\left[\frac{\sigma}{2 B L}\right]+2\right)$.

We have assumed that $\frac{|j(A)|}{|I(A)|} \geqq \frac{1}{2}$ in Proposition 1 - it is an immaterial restriction, since our aim will be to prove that this ratio cannot be too close to 1 . We shall not explicitly verify this when using Proposition 1 in the future.

\section{Periodic Orbits of the Critical Point}

In this section we shall study periodic orbits of the critical points for maps $f_{t}$ with $t$ in $I(A, k)$, moreover with $\varrho(t) \in A$. Our objective is to find a sense in which these orbits are geometrically far from those with rotation numbers $\frac{p}{q}$ or $\frac{p^{\prime}}{q^{\prime}}$.

It will be usually more natural to consider things on the circle instead of the real line. A map $f_{t}$ then projects to a circle homeomorphism, denoted by $G$. Lengths of arcs, their ratios and cross-ratios have the obvious meaning on the circle. Our main tool will be the Cross-Ration Inequality. Observe that constants $C_{k}$ are independent of the particular choice of $G$. All other constants found in this section are of this type, which we shall mark by referring to them as "universal constants." As the result, our estimations are independent of $A$.

The Farey interval $A$ is assumed to be a good one. We shall use letters $p, q, p^{\prime}, q^{\prime}$, $r, s$ in the sense defined in previous sections with the convention $q^{\prime}>q$. Images of a point by iterates of $G$ will be written as the point marked with the appropriate superscript - for example $z^{q}$ for $G^{q}(z)$. The critical point will be denoted by $z$.

We assume that $G$ is chosen so that $z$ is periodic with rotation number of the form $u_{n}=\frac{n p+p^{\prime}}{n q+q^{\prime}}$ for some natural $n$. The geometric shape of the resulting orbit is shown on Fig. 1. We also furnish Fig. 2, which illustrates the situation for the rotation number of the form $v_{n}=\frac{p+n p^{\prime}}{q+n q^{\prime \prime}}$, however we shall not solve that case explicitly, but reduce it to the situation with $u$. It is possible that orientations on both figures should be actually reversed. 
Fig. 1

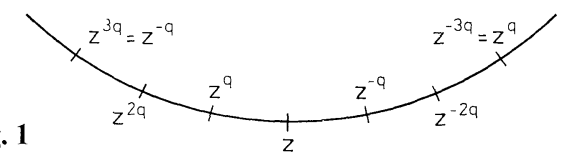

Fig. 2
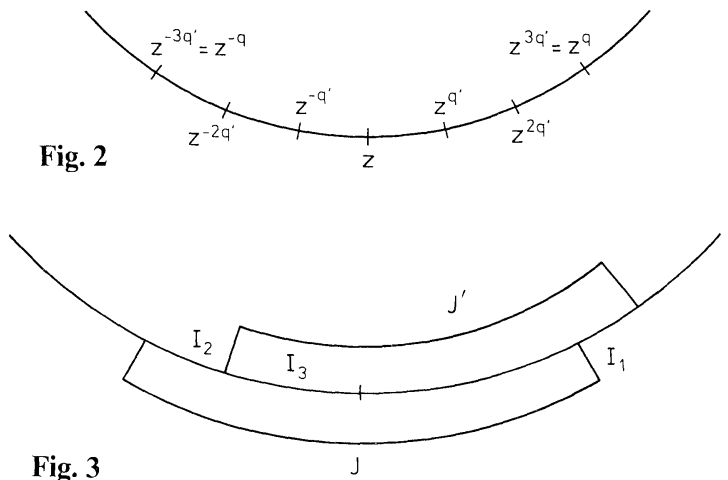

We consider intervals with endpoints at neighbour points of the orbit of $z$. The set of such intervals is denoted by $X$. In other words, $X$ consists of images by iterates of $G$ of the $\operatorname{arc}\left(z^{q}, z\right)$. The maps $G$ induces a permutation of $X$.

We fix the orientation of the circle by the demand that $\left(z, z^{s}\right)$ should be positively oriented.

Lemma 8. Let $I_{1}$ and $I_{2}$ be different arcs from $X$ having a common endpoint. There is a universal constant $K_{8}$ with $K_{8}^{-1} \leqq \frac{\left|I_{1}\right|}{\left|I_{2}\right|} \leqq K_{8}$.

Proof. Let us consider $I_{1}$ and $I_{2}$ as in the hypothesis, such that the ratio of lengths $\frac{\left|I_{2}\right|}{\left|I_{1}\right|}$ is minimal. We shall denote that ratio by $S$. Let $a, b, c, d$ be 4 consecutive in the sense of the ordering on the circle points of the orbit of $z$, such that $b$ and $c$ are endpoints of $I_{1}$. Obviously we have $\operatorname{Cr}(a, b, c, d) \leqq S$. There is also $i$ less than the cardinality of $X$, such that $\left(b^{i}, c^{i}\right)$ is the shortest interval of $X$, which implies that $\operatorname{Cr}\left(a^{i}, b^{i}, c^{i}, d^{i}\right) \geqq \frac{1}{4}$. We may choose minimal such $i$ and then, since $H$ induces the permutation of $X$, we may also use C-RI with $k=3$. Thus $K_{8}=\underline{4} \cdot C_{3}$ will work.

Lemma 9. Let $J$ be an arc of the form $\left(z^{i}, z^{i+s}\right)$ for some $i$ and let $J^{\prime}$ be $J$ shifted by one interval from $X$, as shown on Fig. 3. Then $K_{9}^{-1} \leqq \frac{\left|J^{\prime}\right|}{|J|} \leqq K_{9}$ with $K_{9}$ a universal
constant.

Proof. The arc $J$ contains at least 2 arcs from $X$, as provided by the properties of Farey intervals. Thus we obtain in notations of Fig. 3:

$$
\frac{\left|J^{\prime}\right|}{|J|} \geqq \frac{\left|J^{\prime}\right|-\left|I_{1}\right|}{|J|}=1-\frac{\left|I_{2}\right|}{|J|} \geqq 1-\frac{\left|I_{2}\right|}{\left|I_{2}\right|+\left|I_{3}\right|} \geqq 1-\frac{1}{1+K_{8}^{-1}} .
$$


Since $\frac{|J|}{\left|J^{\prime}\right|}$ can be estimated in the same way, we may put $K_{9}^{-1}$ equal to that constant.

Lemma 10. Let us consider intervals $J$ and $J^{\prime}$ of the form $J=\left(z^{i}, z^{i+s}\right)$ and $J^{\prime}=\left(z^{i+s}, z^{i+2 s}\right)$ for some $i$. There is a universal constant $K_{10}$ with $K_{10}^{-1} \leqq \frac{\left|J^{\prime}\right|}{|J|} \leqq K_{10}$.

Proof. The present lemma is analogous to Lemma 8 and the idea of the proof is also similar. We pick $J^{\prime}$ and $J$ like in the hypothesis and with $\frac{|J|}{\left|J^{\prime}\right|}=S$ and minimal. We also choose points $a, b, c, d$ like in the proof of the lemma and make $j$ be minimal such that $\left(b^{j}, c^{j}\right)$ is the shortest of intervals $(b, c), \ldots,\left(b^{q-1}, c^{q-1}\right)$ - of course $j<q$. The first observation is that by Lemma 7a the number $k$ from CR-I for those intervals is 3. Another point is that they constitute "almost a partition" of the circle - it would actually be a partition if the period of $z$ were $q$ or, in other words, if $\left(b^{q-s}, c^{q-s}\right)=\left(b^{q-s}, b^{q}\right)$ were shifted by 1 arc from $X$. Thus, if $j$ is different from both 0 and $q-s$, the argument of Lemma 8 applies - otherwise it works after shifting $(b, c)$ or $\left(b^{q-s}, c^{q-s}\right)$ by 1 arc from $X$. We may put $K_{10}=K_{9} \cdot K_{8}$ in any case.

Lemma 11. Let us consider points $z^{i+q}, z^{i}, z^{s+q+i}, z^{s+i}$ for some $i$. Let $j \leqq q$. Then

$$
D\left(z^{i+q}, z^{i}, z^{i+q+s}, z^{i+s} ; G^{j}\right) \geqq K_{11}
$$

with $K_{11}$ being a uniform constant.

Proof. We denote $z^{i+q}=a, z^{i}=b, z^{i+q+s}=c, z^{s+i}=d$. Let $D\left(a, b, c, d ; G^{j}\right)=S$. By CR-I and Lemma 7, we obtain that $D\left(a^{j}, b^{j}, c^{j}, d^{j} ; G^{q-j}\right) \leqq C_{3}$, thus $D\left(a, b, c, d ; G^{q}\right)$ $\leqq S \cdot C_{3}$. The points $a^{q}, b^{q}, c^{q}, d^{q}$ are shifted by 1 arc from $X$ with respect to $a, b, c, d$. By the above inequality we obtain that either

$$
\frac{\left|\left(a^{q}, b^{q}\right)\right|}{\left|\left(a^{q}, c^{q}\right)\right|} \leqq \sqrt{S \cdot C_{3}} \cdot \frac{|(a, b)|}{|(a, c)|}
$$

or

$$
\frac{\left|\left(c^{q}, d^{q}\right)\right|}{\left|\left(b^{q}, d^{q}\right)\right|} \leqq \sqrt{S \cdot C_{3}} \cdot \frac{|(c, d)|}{|(b, d)|} .
$$

We are going to consider the first case only, because the second one is analogous. Since $\frac{\left|\left(a^{q}, c^{q}\right)\right|}{|(a, c)|} \leqq K_{9}$ by Lemma 9, we obtain

$$
\left|\left(a^{q}, b^{q}\right)\right| \leqq \sqrt{S \cdot C_{3}} \cdot K_{9} \cdot|(a, b)|
$$

and, by Lemma $8, K_{8}^{-1} \leqq \sqrt{S \cdot C_{3}} \cdot K_{9}$. Hence we may put $K_{11}=\frac{1}{K_{8}^{2} \cdot K_{9}^{2} \cdot C_{3}}$.

Now we want to make a comment. Lemma 11 reveals surprising rigidity of action of $G$ on arcs from $X$. Of course, we have also the inequality with the sense 
opposite to that in Lemma 11, as provided by CR-I and Lemma 7. It is clear that such rigidity must impose restrictions on $G$ and $X$ near the critical point which usually distorts cross-ratios by large amounts. Such restriction is proved in Lemma 12. It is worth noticing that the proof of that lemma is the only point in the whole work, where we need existence of a critical point.

Lemma 12. There is a universal constant $K_{12}$ with $\frac{\left|\left(z^{q}, z^{0}\right)\right|}{\left|\left(z^{q}, z^{s+q}\right)\right|} \geqq K_{12}$.

Proof. We know by Lemma 11 that $D\left(z^{q}, z^{0}, z^{q^{\prime}}, z^{s} ; G\right) \geqq K_{11}$. By Lemma 2

$$
D_{h}\left(z^{s}, z^{q^{\prime}}, z^{0} ; G\right) \geqq K_{2} .
$$

It follows that $D_{h}\left(z^{q}, z^{0}, z^{q} ; G\right) \geqq K_{2}^{-1} \cdot K_{11}$. By Lemma $1 \mathrm{c}$ we obtain that

$$
\frac{\left|\left(G\left(z^{q}\right), G\left(z^{0}\right)\right)\right|}{\left|G\left(z^{0}, z^{q^{\prime}}\right)\right|} \leqq 2 \cdot\left(\frac{\left|\left(z^{q}, z^{0}\right)\right|}{\left|\left(z^{0}, z^{q^{\prime}}\right)\right|}\right)^{l}
$$

where $l$ is of the order of the critical point. It follows that lengths of $\left(z^{q}, z^{0}\right)$ and $\left(z^{0}, z^{q^{\prime}}\right)$ are comparable by a universal constant, which implies the claim of the lemma. QED

Lemma 13. There is a universal constant $K_{13}$ such that

$$
\operatorname{Cr}\left(z^{q}, z^{0}, z^{q+2 s}, z^{2 s}\right) \geqq K_{13} .
$$

Proof. By Lemma 10 we obtain

$$
\frac{\left|\left(z^{q^{\prime}}, z^{s}\right)\right|}{\left|\left(z^{q^{\prime}}, z^{s+q^{\prime}}\right)\right|} \leqq K_{10} \cdot \frac{\left|\left(z^{q^{\prime}}, z^{s}\right)\right|}{\left|\left(z^{0}, z^{s}\right)\right|} .
$$

Multiplying both sides by $\frac{\left|\left(z^{q+2 s}, z^{2 s}\right)\right|}{\left|\left(z^{s}, z^{2 s}\right)\right|}$, and using Lemma 11 we get

$$
K_{11} \leqq K_{10} \cdot \frac{\left|\left(z^{s+q}, z^{s}\right)\right|}{\left|\left(z^{0}, z^{s}\right)\right|} \cdot \frac{\left|\left(z^{q+2 s}, z^{2 s}\right)\right|}{\left|\left(z^{s}, z^{2 s}\right)\right|},
$$

from which we obtain $\frac{\left|\left(z^{q+2 s}, z^{2 s}\right)\right|}{\left|\left(z^{s}, z^{2 s}\right)\right|} \geqq K_{11} \cdot K_{10}^{-1}$. On the other hand, by Lemma 12 $\frac{\left|\left(z^{q}, z^{0}\right)\right|}{\left|\left(z^{q}, z^{s}\right)\right|} \geqq K_{12}$. Finally we observe that by Lemma 10 the lengths of $\left(z^{s}, z^{2 s}\right)$ and $\left(z^{0}, z^{2 s}\right)$ are comparable and the same for $\left(z^{q}, z^{q+s}\right)$ and $\left(z^{q}, z^{q+2 s}\right)$. The lemma follows. QED

Lemma 14. There is a universal constant $K_{14}$ with $\frac{\left|\left(z^{q+s}, z^{s}\right)\right|}{\left|\left(z^{0}, z^{s}\right)\right|} \geqq K_{14}$.

Proof. First we note that $\operatorname{Cr}\left(z^{q-s}, z^{-s}, z^{q+s}, z^{s}\right) \geqq K_{13} \cdot C_{2}^{-1}$. It follows from Lemma 13 by Lemma 6 and the Cross-ratio Inequality. It implies in particular that

$$
\frac{\left|\left(z^{q+s}, z^{s}\right)\right|}{\left|\left(z^{-s}, z^{s}\right)\right|} \geqq K_{13} \cdot C_{2}^{-1}
$$

hence also $\frac{\left|\left(z^{q+s}, z^{s}\right)\right|}{\left|\left(z^{0}, z^{s}\right)\right|} \geqq K_{13} \cdot C_{2}^{-1}$. QED 
We sum up the results of this section in the following proposition:

Proposition 2. If the rotation number of $f_{t}$ is of the form $u_{n}$ and the critical point $z$ is periodic, then $\operatorname{Cr}\left(z^{q+1}, z^{1}, z^{q+s+1}, z^{s+1}\right) \geqq Q_{2}$, for $Q_{2}$ an absolute positive constant. In the similar situation when the rotation number is $v_{n}$, then

$$
\operatorname{Cr}\left(z^{q+1}, z^{q+q^{\prime}+1}, z^{1}, z^{q^{\prime}+1}\right) \geqq Q_{2} .
$$

It should be stressed that $Q_{2}$ is independent of $n$.

Proof. If the rotation number is $u_{n}$ we first note that $\operatorname{Cr}\left(z^{q}, z^{0}, z^{q+s}, z^{s}\right)$ is not small by Lemma 12 compared with Lemma 14. Then the first inequality follows by Lemma 11. In order to prove the second one we consider a good Farey interval with endpoints $\frac{p^{\prime}}{q^{\prime}}$ and $\frac{p+p^{\prime}}{q+q^{\prime}}$ instead of $A$. Numbers $u_{n}$ for the new interval are of the
form

$$
\frac{n p^{\prime}+p+p^{\prime}}{n q^{\prime}+q+q^{\prime}}=\frac{(n+1) p^{\prime}+p}{(n+1) q^{\prime}+q}=v_{n+1} .
$$

Then we obtain $\operatorname{Cr}\left(z^{q^{\prime}+1}, z^{1}, z^{q+q^{\prime}+1}, z^{q+1}\right) \geqq Q_{2}$, but this is written by the assumption that $\left(z, z^{q}\right)$ is positively oriented. If we want to follow the convention that $\left(z, z^{s}\right)$ is positively oriented, we have to reverse the order of points obtaining the second inequality claimed in Proposition 2. QED

\section{How Does a Small Change of Parameter Disturb Orbits of the Critical Point?}

From now on we assume that $A$ is a good normalised Farey interval (see the discussion in Sect. 3). We fix some natural $n$ and the corresponding rotation numbers $u_{n}$ and $v_{n}$. We choose $h$ so that the critical point is periodic for $f_{h}$ with rotation number $u_{n}$ and $e$ so that it is periodic for $f_{e}$ with rotation number $v_{n}$. The notations $E(k, A)$ and $H(k, A)$ will be abbreviated to $E$ and $H$ respectively. We denote

$$
\alpha=\max \left(\frac{H-h}{H-e}, \frac{e-E}{h-E}\right) .
$$

From now on, without mention to the contrary, the function $z$ used to define $g_{i}$ will always be $k$. The main result of this section is the following proposition:

Proposition 3. There is a constant $Q_{3}$ independent of $A$ and $n$ such that

$$
\operatorname{Cr}\left(g_{q^{\prime}+1}(E), g_{q^{\prime}+1}(e), g_{q^{\prime}+1}(h), g_{q^{\prime}+1}(H)\right) \leqq \alpha \cdot Q_{3} .
$$

Proof is quite complicated and will be divided into several lemmas.

Lemma 15. The inequality $(q+s) \cdot|I(A)| \leqq \frac{N}{\sigma}$ is satisfied independently of $A$.

Proof. By Lemma 6 the total of lengths of intervals $g_{1}(I(A)), g_{2}(I(A)), \ldots, g_{q+s}(I(A))$ is at least $(q+s) \cdot|I(A)| \cdot \sigma$. On the other hand, it does not exceed $N$ by Proposition 1. The claim of the lemma easily follows. 
Now we introduce some notations. Images of points in the parameter space by function $g_{i}$ will be denoted by the same letters as points themselves printed in boldface and marked with the superscript $i$, for example $g_{i}(E ; k)=\mathbf{E}^{i}$. Further, let $B$ mean supremum over all parameter values of the derivative $\frac{d g_{1}}{d t}-$ it is finite, since $g_{1}$ is of class $C^{1}$. Finally,

$$
z_{i}=\max \left(D\left(\mathbf{E}^{i}, \mathbf{e}^{i}, \mathbf{h}^{i}, \mathbf{H}^{i} ; f_{e}\right), D\left(\mathbf{E}^{i}, \mathbf{e}^{i}, \mathbf{h}^{i}, \mathbf{H}^{i} ; f_{h}\right)\right)
$$

Lemma 16. For positive $i$ there is an inequality:

$$
\operatorname{Cr}\left(\mathbf{E}^{i}, \mathbf{e}^{i}, \mathbf{h}^{i}, \mathbf{H}^{i}\right) \leqq \alpha \cdot \sigma^{-2} \cdot B \cdot L \cdot 2 \cdot \prod_{j=1}^{i-1} \max \left(1, z_{j},\left(2 \frac{L}{\sigma} \cdot\left(\mathbf{H}^{j}-\mathbf{E}^{j}\right)\right)^{2}\right) .
$$

Proof. The proof will follow by induction with respect to $i$. For $i=1$ it is trivial to obtain $\operatorname{Cr}\left(\mathbf{E}^{1}, \mathbf{e}^{1}, \mathbf{h}^{1}, \mathbf{H}^{1}\right) \leqq \alpha \cdot \sigma^{-1} \cdot B$. Since $\frac{L}{\sigma}$ is greater than 1 , the inequality holds.

Let it hold also for some $i$. We denote $f_{e}\left(\mathbf{E}^{i}\right), f_{e}\left(\mathbf{e}^{i}\right), f_{e}\left(\mathbf{h}^{i}\right), f_{e}\left(\mathbf{H}^{i}\right)$ by $\bar{E}, \bar{e}, \bar{h}, \bar{H}$ respectively. We consider $z_{i}$ as the first approximation of the ratio between

$$
\operatorname{Cr}\left(\mathbf{E}^{i+1}, \mathbf{e}^{i+1}, \mathbf{h}^{i+1}, \mathbf{H}^{i+1}\right) \text { and } \operatorname{Cr}\left(\mathbf{E}^{i}, \mathbf{e}^{i}, \mathbf{h}^{i}, \mathbf{H}^{i}\right) .
$$

Our task now is to estimate how much the actual value of that ratio can exceed $z_{i}$. By the theorem of Lagrange we get $\mathbf{e}^{i+1}-\mathbf{E}^{i+1}=\bar{e}-\bar{E}+\frac{\partial f}{\partial t}\left(E, y_{1}\right)(e-E)$ and

$$
\mathbf{h}^{i+1}-\mathbf{E}^{i+1}=\bar{h}-\bar{E}+\frac{\partial f}{\partial t}\left(E, y_{1}\right)(e-E)+\frac{\partial f}{\partial t}\left(h, y_{2}\right)(h-e)
$$

for suitably chosen $y_{1}$ and $y_{2}$. Thus we obtain

$$
\begin{aligned}
\frac{\mathbf{e}^{i+1}-\mathbf{E}^{i+1}}{\mathbf{h}^{i+1}-\mathbf{E}^{i+1}} & \leqq \max \left(\frac{\bar{e}-\bar{E}}{\bar{h}-\bar{E}}, \frac{\frac{\partial f}{\partial t}\left(E, y_{1}\right)(e-E)}{\frac{\partial f}{\partial t}\left(h, y_{2}\right)(h-e)+\frac{\partial f}{\partial t}\left(E, y_{1}\right)(e-E)}\right) \\
& \leqq \max \left(\frac{\bar{e}-\bar{E}}{\bar{h}-\bar{E}}, \frac{L}{\sigma} \frac{e-E}{h-E}\right) \leqq \max \left(\frac{\bar{e}-\bar{E}}{\bar{h}-\bar{E}}, \frac{L}{\sigma} \cdot \alpha\right) .
\end{aligned}
$$

In further reasonings we assume that the inequality

$$
\frac{\mathbf{e}^{i+1}-\mathbf{E}^{i+1}}{\mathbf{h}^{i+1}-\mathbf{E}^{i+1}} \leqq \frac{\bar{e}-\bar{E}}{\bar{h}-\bar{E}}
$$

is verified - if it is not the case, then obviously

$$
\operatorname{Cr}\left(\mathbf{E}^{i+1}, \mathbf{e}^{i+1}, \mathbf{h}^{i+1}, \mathbf{H}^{i+1}\right) \leqq \frac{L}{\sigma} \cdot \alpha,
$$

and the inequality for $i+1$ holds. 
The estimation of the ratio $\frac{\mathbf{H}^{i+1}-\mathbf{h}^{i+1}}{\mathbf{H}^{i+1}-\mathbf{e}^{i+1}}$ is more complicated. We define numbers $D_{1}, D_{2}, D_{3}$ by

$$
\begin{gathered}
D_{1}=\mathbf{h}^{i+1}-\bar{h}, \\
D_{2}=\mathbf{H}^{i+1}-f_{h}\left(\mathbf{H}^{i}\right), \\
D_{3}=\mathbf{H}^{i+1}-\bar{H}-D_{1}-D_{2} .
\end{gathered}
$$

Observe that $D_{1}$ and $D_{2}$ are necessarily positive, while $D_{3}$ may be negative. The following relations hold:

$$
\mathbf{h}^{i+1}=\bar{h}+D_{1}, \quad \mathbf{H}^{i+1}=D_{1}+D_{2}+D_{3}+\bar{H}, \quad \text { and } \quad \mathbf{e}^{i+1}=\bar{e} .
$$

Using them we compute

$$
\frac{\mathbf{H}^{i+1}-\mathbf{h}^{i+1}}{\mathbf{H}^{i+1}-\mathbf{e}^{i+1}}=\frac{\bar{H}-\bar{h}+D_{2}+D_{3}}{\bar{H}-\bar{e}+D_{1}+D_{2}+D_{3}} \leqq \max \left(\frac{\bar{H}-\bar{h}}{\bar{H}-\bar{e}}, \frac{D_{2}+D_{3}}{D_{1}+D_{2}+D_{3}}\right) \text {. }
$$

It will be enough to bound $\frac{D_{2}+D_{3}}{D_{1}+D_{2}+D_{3}}$, since otherwise we obtain by $(*)$ that

$$
\operatorname{Cr}\left(\mathbf{E}^{i+1}, \mathbf{e}^{i+1}, \mathbf{h}^{i+1}, \mathbf{H}^{i+1}\right) \leqq \operatorname{Cr}(\bar{E}, \bar{e}, \bar{h}, \bar{H}) \leqq z_{i} \cdot \operatorname{Cr}\left(\mathbf{E}^{i}, \mathbf{e}^{i}, \mathbf{h}^{i}, \mathbf{H}^{i}\right),
$$

in which case the inequality for $i+1$ is obviously satisfied. We consider 2 cases:

$1^{\circ} D_{2} \geqq D_{3}$, then we have

$$
\frac{D_{2}+D_{3}}{D_{1}+D_{2}+D_{3}} \leqq 2 \cdot \frac{D_{2}}{D_{1}+D_{2}} \leqq 2 \cdot \frac{L}{\sigma} \cdot \alpha
$$

where the last inequality follows easily from the hypotheses of TV by the mean value theorem. Thus in this case the inequality for $i+1$ is also satisfied.

$2^{\circ} \quad D_{3} \geqq D_{2}$. We calculate $D_{3}$ :

$$
\begin{aligned}
D_{3} & =\mathbf{H}^{i+1}-\bar{H}-D_{1}-D_{2}=\mathbf{H}^{i+1}-\bar{H}-\left(\mathbf{H}^{i+1}-f_{h}\left(\mathbf{H}^{i}\right)\right)-D_{1} \\
& =f_{h}\left(\mathbf{H}^{i}\right)-\bar{H}-\left(f_{h}\left(\mathbf{h}^{i}\right)-\bar{h}\right)=\int_{e}^{h}\left(\frac{\partial f}{\partial t}\left(y, \mathbf{H}^{i}\right)-\frac{\partial f}{\partial t}\left(y, \mathbf{h}^{i}\right)\right) d y \\
& \leqq \int_{e}^{h} L \cdot\left(\mathbf{H}^{i}-\mathbf{h}^{i}\right) d y=L \cdot\left(\mathbf{H}^{i}-\mathbf{h}^{i}\right)(h-e),
\end{aligned}
$$

where $L$ bounds $\left|\frac{\partial^{2} f}{\partial x \partial t}\right|$ on $T \times \mathbb{R}$. Further we obtain

$$
\frac{\mathbf{H}^{i+1}-\mathbf{h}^{i+1}}{\mathbf{H}^{i+1}-\mathbf{e}^{i+1}} \leqq \frac{D_{2}+D_{3}}{D_{1}+D_{2}+D_{3}} \leqq 2 \cdot \frac{D_{3}}{D_{1}} \leqq 2 \cdot \frac{L}{\sigma}\left(\mathbf{H}^{i}-\mathbf{h}^{i}\right) .
$$

Then we may repeat the same reasoning interchanging roles between $H$ and $E$ and between $e$ and $h$, considering $f_{h}(E), f_{h}(e), f_{h}(h), f_{h}(H)$ instead of $\bar{E}, \bar{e}, \bar{h}, \bar{H}$ and carrying out analogous estimations. The result will be that either the inequality for $i+1$ is satisfied or the inequality

$$
\frac{\mathbf{e}^{i+1}-\mathbf{E}^{i+1}}{h^{i+1}-E^{i+1}} \leqq 2 \cdot \frac{L}{\sigma}\left(\mathbf{e}^{i}-\mathbf{E}^{i}\right)
$$


holds. Multiplying both sides by $(* *)$ we get

$$
\operatorname{Cr}\left(\mathbf{E}^{i+1}, \mathbf{e}^{i+1}, \mathbf{h}^{i+1}, \mathbf{H}^{i+1}\right) \leqq\left(2 \cdot \frac{L}{\sigma}\right)^{2} \cdot \operatorname{Cr}\left(\mathbf{E}^{i}, \mathbf{e}^{i}, \mathbf{h}^{i}, \mathbf{H}^{i}\right) \cdot\left(\mathbf{H}^{i}-\mathbf{e}^{i}\right) \cdot\left(\mathbf{h}^{i}-\mathbf{E}^{i}\right) .
$$

The inequality for $i+1$ follows. QED

Proof of Proposition 3. From Lemma 16 we obtain in particular that

$$
\operatorname{Cr}\left(\mathbf{E}^{q^{\prime}+1}, \mathbf{e}^{q^{\prime}+1}, \mathbf{h}^{q^{\prime}+1}, \mathbf{H}^{q^{\prime}+1}\right) \leqq \frac{2 B L \alpha}{\sigma^{2}} \cdot \prod_{j=1}^{q^{\prime}} \max \left(1, z_{j},\left(2 \cdot \frac{L}{\sigma}\left(\mathbf{H}^{j}-\mathbf{E}^{j}\right)\right)^{2}\right) .
$$

We need to prove that the right-hand side of this inequality is bounded by a uniform constant. By Proposition 1 and the Cross-ratio Inequality we obtain that

$$
\prod_{j=1}^{q^{\prime}} \max \left(1, z_{j}\right) \leqq C_{N}^{2}
$$

(we need the square since $z_{j}$ are distortions of cross-ratios for 2 different maps $-f_{e}$ and $f_{h}$ ). Further we use

$$
\max \left(1,2 \cdot \frac{L}{\sigma}\left(\mathbf{H}^{j}-E^{j}\right)\right) \leqq \begin{cases}1 & \text { if } \quad \mathbf{H}^{j}-\mathbf{E}^{j} \leqq \frac{\sigma}{2 L} \\ 2 \cdot \frac{L}{\sigma} & \text { otherwise. }\end{cases}
$$

By Proposition 1 the second possibility can occur for at most $\left[\frac{2 L N}{\sigma}\right]$ numbers $j$.
The proposition follows. QED

Even if Proposition 3 holds, we emphasize that functions $g$ have not, as a rule, a negative Schwarzian derivative, even if the functions $f_{t}$ are quite nice and depend on the parameter in a simple way. Hence it would not be enough to assume that the cross-ratio $\operatorname{Cr}(E, e, h, H)$ is small in order to obtain the claim of Proposition 3.

\section{Why $\propto$ Cannot be Too Small}

We prove in this section that Proposition 2 implies that the number $\alpha$ of the previous section cannot be very small. In merging the results of Sect. 5, which was done on the circle, and the rest of our work, which applies to the situation lifted on the real line, we meet some notational problem. We introduce the following convention: $X^{0}=g_{1}(X ; k)$ where $X$ should be replaced with $E, e, h, H$,

$$
\begin{gathered}
X^{-s}=g_{1-s}(X ; k)+r, \\
X^{s}=g_{s+1}(X ; k)-r, \\
X^{q^{\prime}}=g_{q^{\prime}+1}(X ; k)-p^{\prime}, \\
X^{q}=g_{q+1}(X ; k)-p .
\end{gathered}
$$

Using the new notation we need not to trouble whether we use points of the circle or from the real line. Please note, however, that points with superscript 0 mean critical values now. 
Lemma 17. The following inequalities are satisfied:

$$
\left|\left(e^{q}, e^{0}\right)\right| \geqq \sigma \cdot(h-e) \quad \text { and } \quad\left|\left(h^{0}, h^{q^{\prime}}\right)\right| \geqq \sigma \cdot(h-e) .
$$

Proof. We shall concentrate on the proof of the first inequality only, since the proof of the second one is quite analogous. We consider the function $G: T \rightarrow S^{1}$ defined by $G(t)=g_{q+1}(t ; k(e))-p$, where $k(e)$ means the constant function. Then we have:
a) $G(e)=e^{q}$,
b) $G(h)<e^{0}-$ it is so because $\varrho(h)<\frac{p}{q}$,
c) $\frac{d G}{d t} \geqq \sigma$ by Lemma 6 .

The lemma follows immediately. QED

By Proposition 2 the sizes of $\left(e^{q}, e^{0}\right)$ and $\left(e^{0}, e^{q^{\prime}}\right)$ are comparable, as are the sizes of $\left(h^{q}, h^{0}\right),\left(h^{0}, h^{q^{\prime}}\right),\left(h^{q^{\prime}}, h^{s}\right)$.

All possible ratios between them are bounded by $Q_{2}^{-1}$. There is also an obvious relation $\left|\left(e^{0}, h^{0}\right)\right| \leqq L \cdot(h-e)$. From this and Lemma 17 we obtain further

$$
\left|\left(e^{q^{\prime}}, h^{q^{\prime}}\right)\right| \leqq\left|\left(e^{0}, h^{0}\right)\right|+\left|\left(h^{0}, h^{q^{\prime}}\right)\right| \leqq\left(1+\frac{L}{\sigma}\right) \cdot\left|\left(h^{0}, h^{q^{\prime}}\right)\right| .
$$

Using now the corollary from Proposition 2 we get

$$
\left|\left(h^{q^{\prime}}, h^{s}\right)\right| \geqq Q_{2} \cdot\left|\left(h^{0}, h^{q^{\prime}}\right)\right| \geqq Q_{2} \cdot\left(1+\frac{L}{\sigma}\right)^{-1}\left|\left(e^{q^{\prime}}, h^{q^{\prime}}\right)\right| .
$$

On the other hand we have $\left|\left(e^{q}, h^{0}\right)\right| \geqq\left|\left(h^{q}, h^{0}\right)\right|$, because $h^{q}>e^{q}$. Hence

$$
\left|\left(e^{0}, e^{q^{\prime}}\right)\right| \geqq Q_{2}\left|\left(e^{q}, e^{0}\right)\right| \geqq Q_{2}\left(1+\frac{L}{\sigma}\right)^{-1}\left|\left(e^{q}, h^{0}\right)\right|
$$

- the last inequality is implied by Lemma 17 . Further we get

$$
\left|\left(h^{q}, h^{0}\right)\right| \geqq Q_{2}\left|\left(h^{0}, h^{q^{\prime}}\right)\right| \geqq Q_{2} \cdot\left(1+\frac{L}{\sigma}\right)^{-1}\left|\left(e^{q^{\prime}}, h^{q^{\prime}}\right)\right|
$$
by $(*)$. It follows that the ratio $\frac{\left|\left(e^{0}, e^{q^{\prime}}\right)\right|}{\left|\left(e^{q^{\prime}}, h^{q^{\prime}}\right)\right|}$ is bounded away from 0 by an absolute
constant.

Recalling also (**), we see that $\operatorname{Cr}\left(e^{0}, e^{q^{\prime}}, h^{q^{\prime}}, h^{s}\right) \geqq Q_{7}$ with $Q_{7}$ being a positive absolute constant. The more $\operatorname{Cr}\left(E^{0}, e^{q^{\prime}}, h^{q^{\prime}}, H^{s}\right) \geqq Q_{7}$. Now Proposition 3 implies that $\alpha \geqq Q_{7} \cdot Q_{3}^{-1}$.

\section{Completion of the Proof}

Since the lower bound for $\alpha$ obtained in the previous section is independent of $n$, the result of the previous section may be stated as follows:

For any good Farey interval $A$ a part of $I(A)$ consisting of not less than $Q_{7} \cdot Q_{3}^{-1}$ of its whole length is occupied by parameter values corresponding to rational rotation numbers. 
Suppose now that the set $\varrho^{-1}(\mathbb{R} \backslash \mathbb{Q})$ has a positive Lebesgue measure. Then it contains a point of density $y$. There exists a descending sequence $A_{n}$ of good Farey intervals such that $\bigcap_{n=1}^{\infty} A_{n}=\{\varrho(y)\}$. Then also $\bigcap_{n=1}^{\infty}\left(A_{n}\right)=\{y\}$, since every irrational rotation number corresponds to only 1 parameter value, as follows for example from Lemma 6 and the density of orbits of a homeomorphism with an irrational rotation number proved in [7]. We see that $y$ is not a point of density of $\varrho^{-1}(\mathbb{R} \backslash \mathbb{Q})$, hence the Lebesgue measure of the latter is 0 .

\section{References}

1. Hardy, G.H., Wright, E.M.: An introduction to the theory of numbers. Oxford: Clarendon Press 1945, Chap. X

2. Herman, M.: Mesure de Lebesgue et nombre de rotation, Geometry and Topology. Lecture Notes in Mathematics, Vol. 597, pp. 271-293. Berlin, Heidelberg, New York: Springer 1977

3. Jensen, M., Bak, P., Bohr, T.: Complete Devil's staircase, fractal dimension and universality of mode-locking structure in the circle map. Phys. Rev. Lett. 50, 1637-1639 (1983)

4. de Melo, W., van Strien, S.: A structure theorem in one dimensional dynamics (preprint)

5. Preston, C.: Iterates of maps on an interval. Lecture Notes in Mathematics, Vol. 999. Berlin, Heidelberg, New York: Springer 1983

6. Światek, G.: Endpoints of rotation intervals for maps of the circle (preprint)

7. Yoccoz, J.C.: Il n'y a pas de contre-exemple de Denjoy analitique. C.R. Acad. Sci. Paris, t. 298, Serue I, n'7 (1984)

Communicated by J.-P. Eckmann

Received March 23, 1987; in revised form January 4, 1988 